\title{
COMPARISON BETWEEN ENERGY EFFICIENT COOPERATIVE MIMO AND COOPERATIVE RELAY IN ENERGY CONSTRAINED WSN
}

\author{
Nidhi Chuhan ${ }^{1}$ and Mayank Sharma ${ }^{2}$ \\ ${ }^{1,2}$ Department of Electronics and Communication Engineering, \\ 1Jaypee University of information Technology, Solan, India \\ ${ }^{2}$ ITM UNIVERSITY, Gwalior, India
}

\begin{abstract}
Wireless sensor network consists of thousand number of sensor nodes employed in a wide range of data gathering application such as environmental gathering, military applications etc. Each sensor node in WS $N$ are powered by battery having limited energy so it is very difficult to replace there batteries for prolonged the network life time. Maximizing the network lifetime have been most important design goal for the network. In WSN channel fading and radio interference constitute a big dispute in design of energy efficient communication protocol. To reduce fading and energy consumption in WSN, MIMO MISO SISO scheme is employed but implement multiple antenna technology to sensor network is improbable because of the sensor node generally support a single antenna. Cooperative scheme are utilized in WSN by applying the collective nature of the sensor nodes to provide reliable communication links in order to lower the total energy consumption. In this paper, the cooperative technique are inspected and by comparing different cooperative techniques we can elect the best cooperative scheme for energy constrained WS N application.
\end{abstract}

\section{KEYWORDS}

Wireless sensor network, cooperative relay, cooperative MISO, energy consumption.

\section{INTRODUCTION}

For most application sensor nodes depends on battery for their power supply hence battery decay may have a great impact on the overall network performance. Rather of using a contend approach such as in traditional wireless network for energy conservation, sensor nodes are supposed to cooperate to prolonged the network life time[2][3]. Multiple sensor nodes cooperate to transmit and receive and such a configuration is known as cooperative technique. This technique reduce both fading and transmission energy. Cooperative technique [1] can be classified as cooperative MIMO, MISO, SISO and cooperative relay. In a cooperative relay technique, network uses the neighbor nodes to cooperate the source by transmitting the source information to the destination for achieving spatial diversity. Thus the conclusion is that system performance is improved and less transmission energy is required. Multi-antenna system have been studied exclusively

In Recent Year Due To their Potential To Greatly Increase The Channel capacity In fading ,Environment .Due To Limited Physical Size of Sensor node .Direct application of multi antenna

DOI: $10.5121 /$ jant.2016.2202 
to sensor Network is impractical .A cooperative MIMO scheme can be constructed if we allow individual single antenna node to cooperate on information transmission and reception. Cooperative MIMO [4] technique aid exploits the diversity gain in order to reduce energy consumption. In this paper we inspected the energy consumption of cooperative MIMO and cooperative relay [6]. By comparing these two techniques we can conclude that cooperative MIMO perform better than the cooperative relay but under certain condition cooperative relay is better than the cooperative MIMO. When some information has to be transmitted from source to destination the efficient solution is depend on the position sensor nodes. If the sensor nodes are present in the middle means at equal distance from source and destination cooperative relay is preferred but for long distance between transmitter and receiver cooperative MIMO is best solution.

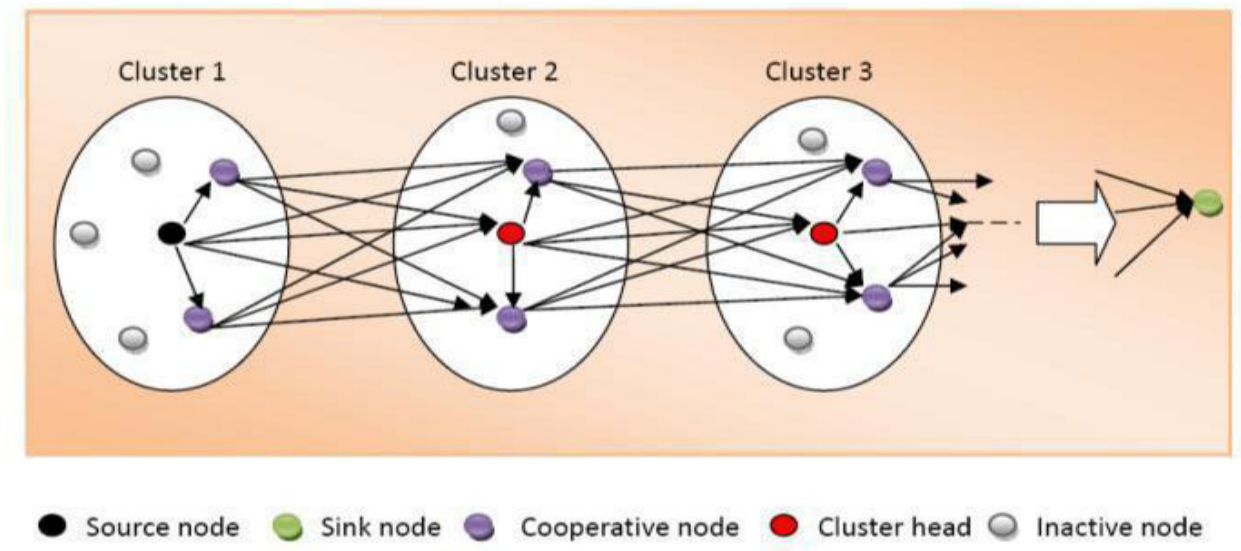

Fig.1 cooperative MIMO

In fig. 1 cooperative nodes are cooperate with the source node by transmitting source information to other clusters in the network and finally transmit it to the destination. Nodes other than cooperative are in sleep mode. Fig. 2 represents the cooperative relay where cooperative node are present at the middle and cooperate with source node by transmitting its information to the destination. With the same diversity gain MIMO performance is always higher than the cooperative relay.

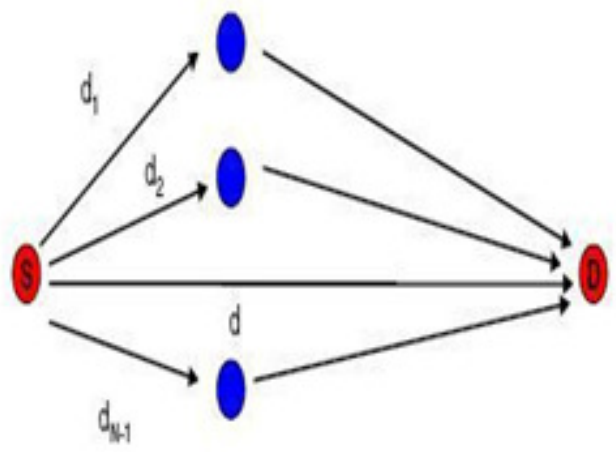

Fig. 2 cooperative relaying 
Remainder of this paper is organized as follows: In section II, non-cooperative energy consumption model. In section III, comparing two cooperative scheme on the basis of energy consumption model. Section IV summaries our conclusion.

\section{ENERgy CONSUMPTION MODEL}

\subsection{Non -cooperative communication system}

For energy calculation of cooperative system we used the energy model with same parameter as in [11]. Let us consider a non-cooperative MIMO system with $\mathrm{N}_{t}$ transmit and $\mathrm{M}_{\mathrm{r}}$ received antennas, respectively. The total power consumption of non-cooperative RF system based on two components: circuit power consumption $\left(\mathrm{P}_{\mathrm{cir}}\right)$ and power consumption of the power amplifier $(\mathrm{Pa}$ $\mathrm{mp})$.
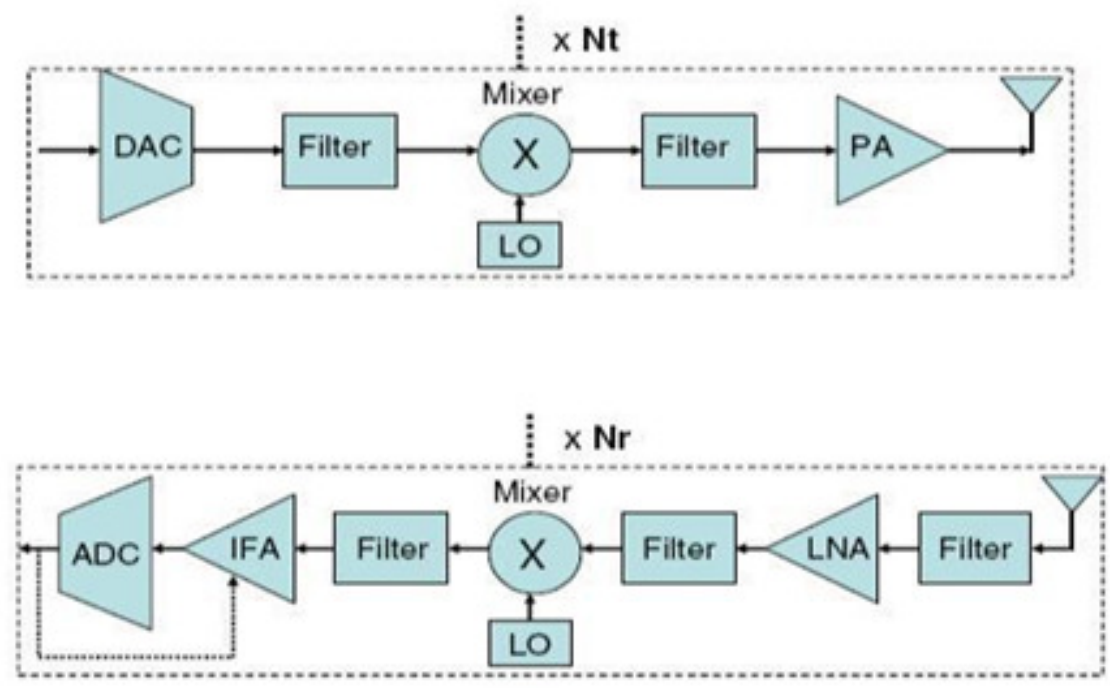

Fig.3 Transmitter and Receiver block

In the channel is square law path loss then the power consumption of the power amplifier is depends on the transmit power which is given by the link budget equation such as [7]

$$
P_{T x}=\frac{E_{b} R_{b} \times(4 \pi d)^{2} M_{l} M_{f}}{G_{t} G_{r} \lambda^{2}}
$$

Where $E_{b}$ is the required energy per bit, $R_{b}$ is the bit rate, $d$ is the transmission distance between transmitter and receiver, $G_{t}$ and $G_{r}$ antenna gain, $\lambda$ is the carrier wavelength, $M_{1}$ is the link margin, $\mathrm{N}_{\mathrm{f}}$ is the noise figure. Therefore Power consumption of power amp lifier can be calculated as

$$
P_{a m p}=(1+\alpha) P_{t x}
$$




$$
\text { Where }{ }^{\alpha=\frac{\varepsilon}{\eta}-1} \text { with } \xi \text { drain efficiency of power amplifier }
$$

and ndenotes power to average ratio (PAR) which rely on modulation scheme used and constellation size. The total circuit power consumption is given as

$$
P_{\text {cir }}=N_{t}\left(P_{D A C}+P_{M I X}+P_{s y n}+P_{\text {filt }}\right)+N_{r}\left(P_{L N A}+P_{M I X}+P_{I F A}+P_{f i l r}+P_{A D C}+P_{s y n}\right)
$$

Where $P_{\text {Dac, }}$ P $_{\text {mix }}$ Psyn, Pfilt, Plna, PIfA, Pfilr, PAdC denote Power Consumption value OF DAC ,Mixer ,filter ,low noise amplifier, If amplifier ADC Whose Value presented In table Therefore Total Power Consumption For a non - Cooperative System is given By

$$
E_{\text {total }}=\left(P_{a m p}+P_{\text {cir }}\right) / R_{b}
$$

\subsection{Cooperative MIMO System}

The extra energy of the native cooperative data exchange is dependent on the number of cooperative antennas and also on local inter-node distance between two nodes at both Tx and Rx side. In case of cooperative MIMO, if we want to transmit $\mathrm{N}_{\mathrm{b}}$ bits from source to destination. Then source must firstly broadcast transmit bits to $\mathrm{N}_{\mathrm{t}}-1$ cooperative nodes. Based on the SISO non-cooperative model energy consumption for local data exchange phase is calculated as $\mathrm{E}_{\text {CoopTx. }}$ At the receiver cluster $M_{r}-1$ cooperative nodes firstly receive MIMO modulated symbols quantize one STBC symbol to $\mathrm{N}_{\mathrm{sb}}$ bits and the retransmit their quantize bits to respectively to the destination node using un-coded SISO 16-QAM modulation. Therefore transmission in cooperative MIMO takes place in two communication phases: one is data exchange phase and other one is MIMO transmission phase. The total energy consumption of cooperative MIMO technique is

$$
E_{\text {coopMISO }}=E_{\text {coop }} T_{x}+E_{a m p}+E_{\text {cir }}+E_{\text {coop }} R_{x}
$$

Where $\mathrm{E}_{\mathrm{Coop} T \mathrm{x}}$ is extra cooperative energy consumption in the transmitter side and depend on the energy consumption per bit

Which is calculated as

$$
E_{\text {coop }} T_{x}=N_{b} E_{p b c o o p} T_{x}
$$

and $\mathrm{E}_{\mathrm{CoopRx}}$ is given as,

$$
E_{\text {coop }} R_{x}=N_{s b}\left(M_{r}-1\right) N_{b} E_{p b c o o p} R_{x}
$$




\subsection{Cooperative Relay System}

For the cooperative relay system total energy consumption is the sum of energy consumption of each transmission phase. So the total energy consumption of relay system is given as

$$
E_{\text {relay }}=\sum_{i=1}^{N} E_{\text {total }}(i)
$$

\section{Figures and Tables}

Table 1. System Parameter

\begin{tabular}{|c|c|}
\hline $\mathrm{F}_{\mathrm{c}}=2.5 \mathrm{GHz}$ & $\eta=0.35$ \\
& \\
$\mathrm{G}_{\mathrm{t}} \mathrm{G}_{\mathrm{r}}=5 \mathrm{dBi}$ & $\mathrm{N}_{0} / 2=-174 \mathrm{~d} \mathrm{Bm}$ \\
$\mathrm{B}=10 \mathrm{kHz}$ & $\beta=1$ \\
& $\mathrm{P}_{\mathrm{Syn}}=50 \mathrm{Mw}$ \\
$\mathrm{P}_{\mathrm{MIX}}=30.3 \mathrm{~mW}$ & $\mathrm{~T}_{\mathrm{S}}=1 / \mathrm{B}$ \\
$\mathrm{P}_{\mathrm{b}}=10^{-3}$ & $\mathrm{P}_{\mathrm{LNA}}=20 \mathrm{~mW}$ \\
Pfilt $=\mathrm{Pfil}=2.5 \mathrm{mw}$ & $\mathrm{N}_{\mathrm{l}}=40 \mathrm{~dB}$ \\
$\mathrm{~N}_{\mathrm{f}}=10 \mathrm{~dB}$ & \\
\hline
\end{tabular}

\section{Simulation RESUlT}

The circuit consumption of the cooperative relay scheme will be lower than cooperative MIMO at shorter distance because cooperative MIMO needed extra circuit energy consumption. In cooperative relay the distance between destination and relay node are shorter, so that for ensuring the same receiver SNR less transmission energy than MIMO scheme is required. Cooperative MIMO technique needs less required SNR At distance d=400m, 30\% energy can be saved by using cooperative MIMO scheme when Compared with the cooperative relay.

In fig. 5 when we consider three transmission nodes at distance $\mathrm{d}=400 \mathrm{~m}, 42 \%$ energy can be saved by using cooperative MIMO scheme in comparison with the cooperative relay scheme. A cooperative MIMO network in comparison with cooperative relay is better not only in terms of energy consumption but also transmission delay as well. 


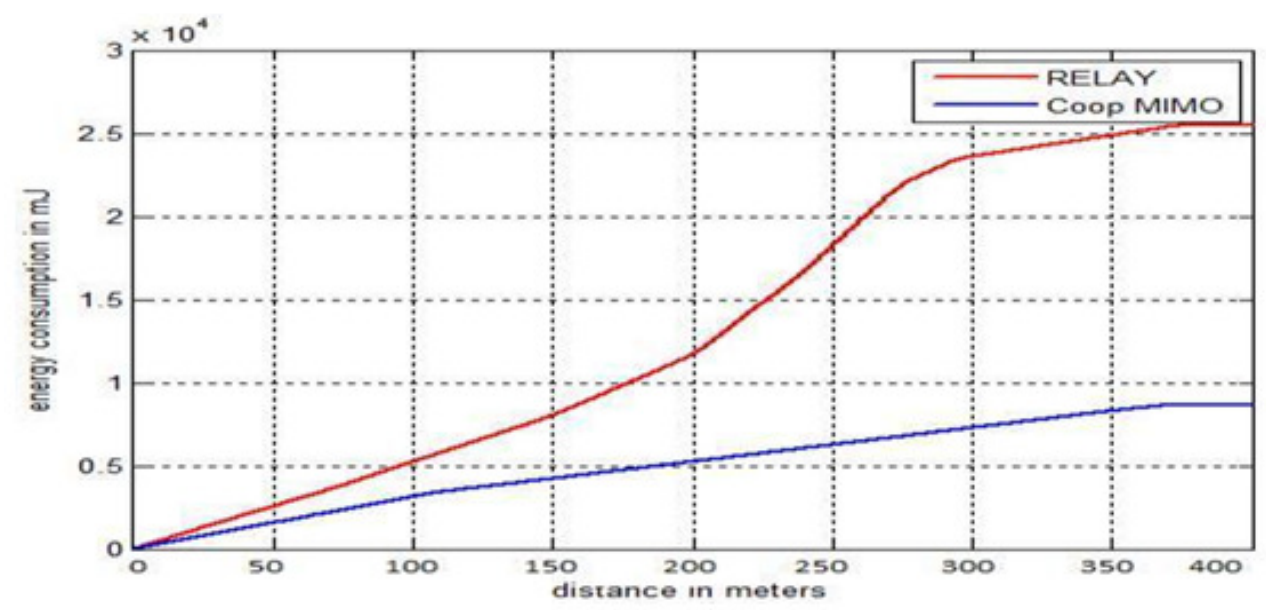

Fig. 4 cooperative MIMO versus cooperative relay $(\mathrm{Tx}=3)]$

\section{Conclusions}

Cooperative scheme contribute agreeable assistance for WSN when temporal and antenna diversity can be exploited to reduce fading as well as transmission energy consumption. Relay scheme are less efficient than the cooperative MIMO in terms of energy consumption at longer distance. For the same SNR relay technique performance is bad as compare to cooperative MIMO. But advantage of relay over cooperative MIMO is that relay technique are not affected by time synchronization error. The performance of relay is better as compare to the cooperative MIMO when transmission synchronization error become significant. Performance and energy consumption comparison with cooperative MIMO with data aggregation will be investigated in future work. Therefore performance of cooperative MIMO is better. The subsequent figure in this paper represent the total energy to transmit $10^{6}$ bits with error rate requirement FER $=10^{-3}$ source sensor node $\mathrm{S}$ to a destination node $\mathrm{D}$.

The total distance between cooperative nodes is $d_{m=} 6 \mathrm{~m}$ and distance between source and relay sensor nodes is $\mathrm{dsr}=\mathrm{d} / 3$. Fig. 4 shows the energy consumption of cooperative MIMO technique in comparison with cooperative relay. When we consider two transmissions node at longer distance energy consumption of cooperative MIMO is lower due to the fact that cooperative MIMO needs less required SNR. 


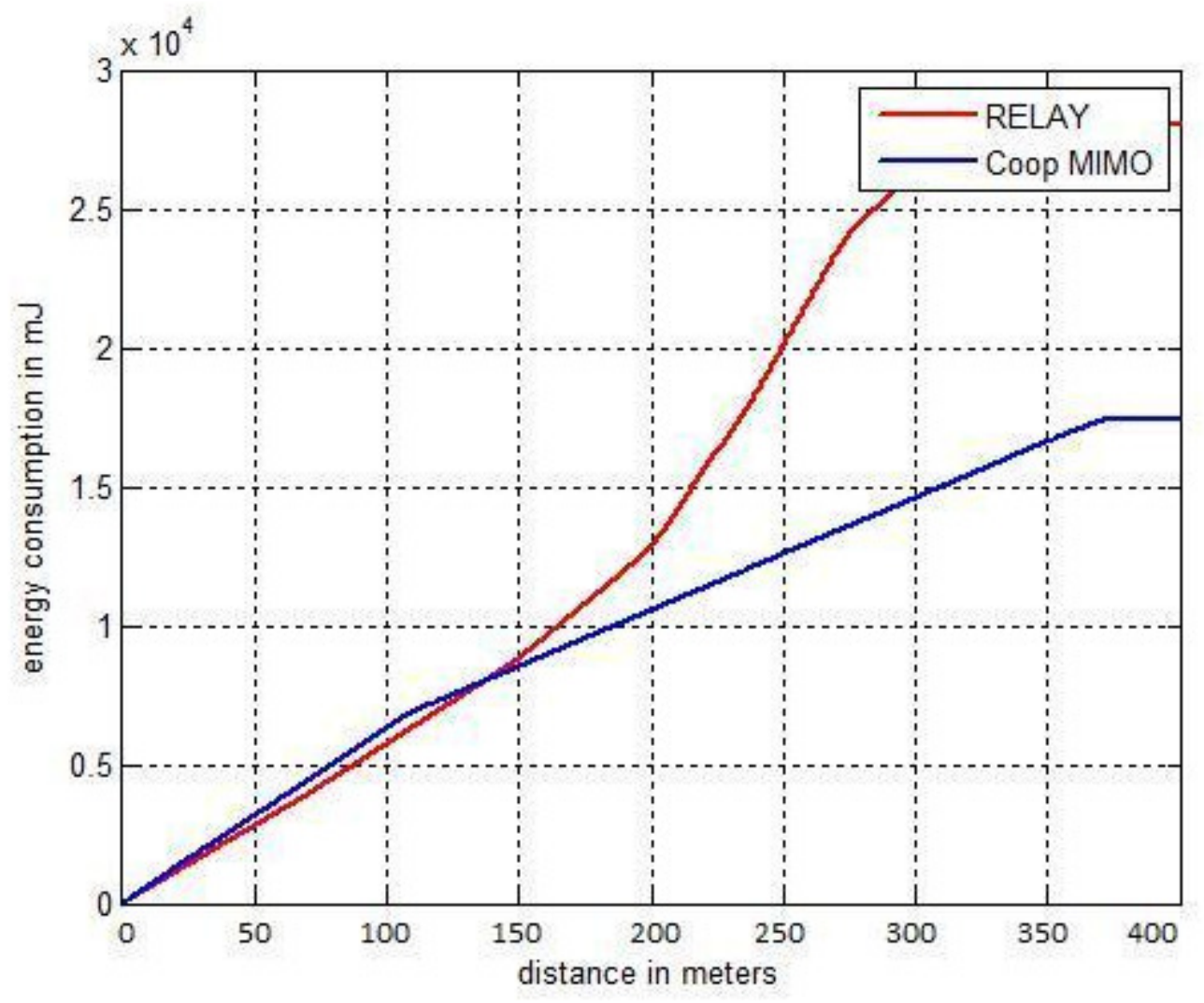

Fig. 5 cooperative MIMO versus cooperative relay $(\mathrm{Tx}=3)$

\section{REFERENCES}

[1] Nosratinia, Aria, Todd E. Hunter, and Ahmadreza Hedayat. "Cooperative communication in wireless networks." Communications Magazine, IEEE 42.10 (2004): 74-80.

[2] Laneman, J.Nicholas, David NC Tse, and Gregory W.Wornell. "Cooperative diversity in wireless networks: Efficient protocols and outage behavior." Information Theory, IEEE Transactions on 50.12 (2004): 3062-3080.

[3] Laneman, J. Nicholas, and Gregory W. Wornell. "Energy-efficient antenna sharing and relaying for wireless networks." In Wireless Communications and Networking Confernce, 2000. WCNC. 2000 IEEE, vol. 1, pp. 7-12. IEEE, 2000.

[4] Nguyen, T. D., Berder, O., \& Sentieys, O. (2007, April). Cooperative MIMO schemes optimal selection for wireless sensor networks. In Vehicular Technology Conference, 2007. VTC2007-Spring. IEEE 65th (pp. 85-89). IEEE

[5] Sendonaris, Andrew, Elza Erkip, and Behnaam Aazhang. "User cooperation diversity. Part I. System description." Communications, IEEE Transactions on 51, no. 11 (2003): 1927-1938.

[6] Proakis, John G. Intersymbol interference in digital communication systems. John Wiley \& Sons, Inc., 2001.

[7] S.K.Jayaweera, "Energy analysis of mimo techniques in wireless sensor networks," in 38th Annual Conference on Information Sciences and Systems, Princeton University, USA, March 2004. 
[8] Li, Xiaohua. "Energy efficient wireless sensor networks with transmission diversity." Electronics Letters 39, no. 24 (2003): 1753-1755.

[9] Sendonaris, Andrew, Elza Erkip, and Behnaam Aazhang. "User cooperation diversity. Part I. System description." Communications, IEEE Transactions on 51, no. 11 (2003): 1927-1938.

[10] Alamouti, Siavash M. "A simple transmit diversity technique for wireless communications." Selected Areas in Communications, IEEE Journal on 16, no. 8 (1998): 1451-1458.

[11] Jagannathan, Sumanth, Hamid Aghajan, and Andrea Goldsmith. "The effect of time synchronization errors on the performance of cooperative MISO systems." In Global Telecommunications Conference Workshops, 2004. GlobeCom Workshops 2004. IEEE, pp. 102-107. IEEE, 2004.

[12] Cui, Shuguang, Andrea J. Goldsmith, and Ahmad Bahai."Modulation optimization under energy constraints." In Communications, 2003. ICC'03. IEEE International Conference on, vol. 4, pp. 28052811. IEEE, 2003.

[13] Cui, Shuguang, Andrea J. Goldsmith, and Ahmad Bahai."Energy-efficiency of MIMO and cooperative MIMO techniques in sensor networks." Selected Areas in Communications, IEEE Journal on 22, no. 6 (2004): 1089-1098.

\section{AuTHORS}

Nidhi Chuhan Received Mtech (ECE) from Jaypee University of information Technology Wakhnaghat solan(H.P)) june 2015.

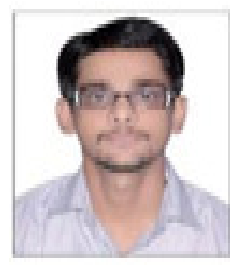

Mayank Sharma was born in india ,MP In 1990, He Received BE (ECE) from ITM UNIVERSE Engineering College Gwalior and Mtech (ECE) from JUIT Wakhnaght. He has 2 year of teaching Experince .1 International and 1 National journal .01 International conference In his Credit .Presently Working as a JRF in ITM university Gwalior. 